\title{
Electrocardiogram Derived Respiration during Sleep
}

\author{
G Dorfman Furman, Z Shinar, A Baharav, S Akselrod \\ Tel-Aviv University, Tel-Aviv, Israel
}

\begin{abstract}
The aim of this study was quantify the ECG Derived Respiration $(E D R)$ in order to extend the capabilities of ECG-based sleep analysis. We examined our results in normal subjects and in patients with Obstructive Sleep Apnea Syndrome (OSAS) or Central Sleep Apnea.

Lead 2 ECG and three measures of respiration (thorax and abdominal effort, and oronasal flow signal) were recorded during sleep studies of 12 normal and 12 OSAS patients. Three parameters, the $R$-wave amplitude (RWA), $R$-wave duration (RWD), and QRS area, were extracted from the ECG signal, resulting in time series that displayed a behavior similar to that of the respiration signals. EDR frequency was correlated with directly measured respiratory frequency, and averaged over all subjects.

The peak-to-peak value of the EDR signals during the apnea event was compared to the average peak-to-peak of the sleep stage, containing the apnea.
\end{abstract}

\section{Introduction}

The gold standard procedure to analyze sleep and diagnosis sleep disorders is a sleep study performed in a sleep laboratory. This procedure is cumbersome and expensive. We have shown in previous studies that sleep can be evaluated by means of Autonomic Nervous System (ANS) function based on Heart Rate Variability (HRV) analysis [1-3].

Respiration during sleep is of major importance since sleep related respiration disorders are frequent and cause important morbidity. There are two distinct ways to monitor respiration: directly, by sampling the air flow, and indirectly, by monitoring the breathing effort from the motion of the thorax or the abdomen.

There are three types of apnea during the night: obstructive, central, and mixed. Of the three, obstructive is the most common. Despite the difference in the etiology of each type, in all three, people stop breathing repeatedly during their sleep, sometimes hundreds of times during the night and often for ten seconds or longer. Obstructive sleep apnea is caused by a blockage of the airway, usually when the soft tissue of the upper airways collapses during sleep. In central sleep apnea, the airway is not blocked but the brain fails to signal the muscles to breathe. Mixed apnea, as the name implies, is a combination of the two. With each apnea event, the brain causes brief arousals and respiration resumes, consequently sleep is extremely fragmented and of poor quality. In this study we deal with the two well defined apnea events: central sleep apnea and obstructive sleep apnea.

The specific aim of this study was to evaluate and compare three different methods to extract respiration from the ECG. The EDR methods are described in the next section. The first two single lead (lead II) methods are based on original algorithms and the third method was developed by Moody et al [4] recording multi-lead ECG. Raymond et al. develop a similar method based on the $\mathrm{T}$ wave for a single lead. The signals were measured during normal respiration and during apnea events in the sleep studies and compared with standard measured respiration previously explained.

\section{Methods \\ 1. Sleep studies}

The study included 24 adult subjects (age 35 $\pm 15,15$ males, and 9 females). All subjects underwent a full standard sleep test, including 4 EEG channels, 2 EOG, 2 EMG, and ECG signal, sampled at $200 \mathrm{~Hz}$. In addition 3 respiration signals were recorded: nasal air flow at 10 $\mathrm{Hz}$, and thorax and abdomen effort at $10 \mathrm{~Hz}$. Sleep stages were determined according to standard Rechtschaffen and Kales $(\mathrm{R} \& \mathrm{~K})$ criteria [5] by a sleep expert. In this work we separate those into Non-REM sleep stages (1-4 stages) and REM sleep stage. The three respiration signals were scanned. Apnea events were detected and marked manually from the visual inspection of all 3 respiratory signals.

\section{EDR extraction}

We evaluated EDR by three methods:

1. Based on the extraction of the $\mathrm{R}$ wave amplitude (RWA) after the subtraction of the ECG baseline.

2. Based on the calculation of the $\mathrm{R}$ wave duration (RWD), defined in [6]. 
3. Based on the area of each normal QRS complex (QRS area) measured over a fixed window width (which is determined by the interval from the PQ junction to the J-point of a normal QRS) [4].

The extracted signals resulted in a time series which display a behavior similar to the standard respiration signals (flow, thorax, and abdomen). The frequency of the signal is proportional to the breathing frequency but its amplitude is not proportional to the respiration volume. An example of the time series behavior is shown in figure 1.

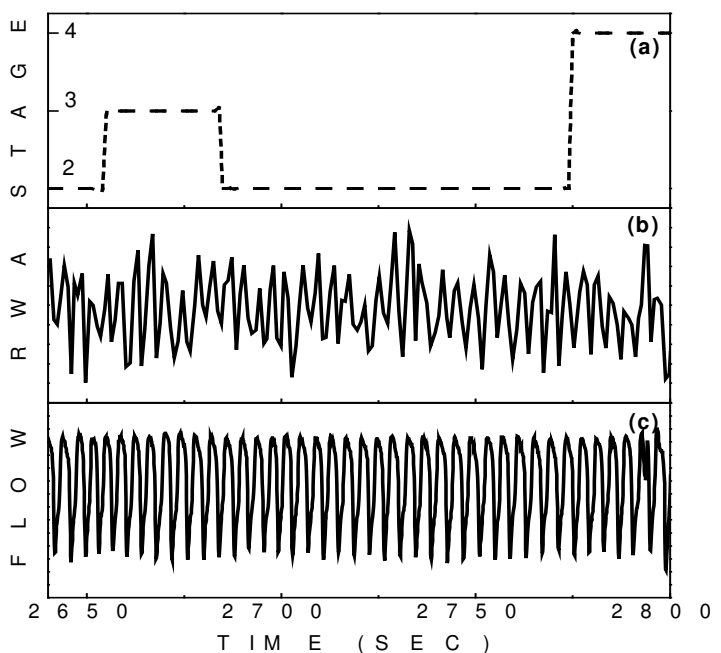

Figure 1. Respiratory behavior during 3 minutes in the night study of a single subject. (a). Sleep 2, 3, and 4 stages occurring during the time series. (b) RWA signal extracted from ECG (a.u.). (c) Respiratory signal (by the air flow) measured directly (a.u.).

\section{EDR evaluation}

\subsection{Frequency Comparison}

The aim of this section was to compare the EDR computerized frequency with calculated frequency from directly measure respiratory signal.

Fourier transform for unevenly sampled data was used to extract EDR frequency from these time series (RWA, RWD, and QRS area). First, a sliding window of 30 points (approximately 30 seconds) from the time series was selected. Second, removing a linear trend by subtracting the straight line that best fits the data in the least squares sense. Third, EDR frequency was extracted by selecting the frequency which corresponds to the maximal power in the spectrum. The overlap between consecutive windows was of 24 points, producing a frequency value every 6 points.
Spectral analysis, using a Fourier transform for evenly sampled data, was applied to the three standard respiratory signals. Calculation was performed using a 30 seconds sliding window with 24 seconds overlap, resulting in a respiration frequency value (for flow, abdomen and thorax signals) every 6 seconds. This value was used as gold standard for comparison with the EDR frequency.

In order to enable comparison between EDR frequency and respiration frequency, an equal time scale was needed. Thus, spline interpolation was used to obtain intermediate points every single second. The resulting interpolated frequency data from the RWA, RWD, and QRS area was correlated to respiratory frequency and averaged over all subjects.

\subsection{Apnea Characterization}

In the next step we looked for changes in EDR signals during apnea events, either obstructive or central. The minimal peak-to-peak value during the apnea events (as extracted from the various EDR methods), was compared to the averaged peak-to-peak of the entire stage episode, containing the apnea event.

The data was first detrended, and the maximal peak-topeak value in each sliding window of 6 second was selected.

\subsection{REM Respiration}

In addition, in order to characterize respiration during the REM stage, based on EDR methods we calculated two predominant parameters: median value and dispersion of each state: REM, NREM and wake. These parameters were calculated for periods of at least 90 seconds, after sleep onset, and averaged over the whole night, and over all subjects.

\section{Results}

\section{EDR Frequency Correlation}

Table 1 shows the resulting correlation between the three measures of respiration frequency (Thorax, Flow and Abdomen) and the three EDR calculated frequencies.

As shown in Table 1, the correlation between the extracted EDR frequency and the directly measure respiratory frequency was above 0.85 .

During the analysis of the respiratory signals, we found in several subjects that some of the collected respiratory data was very noisy (about $10 \%$ of the data). Including this data in the analysis the correlation frequency was above 0.85 . 
Table 1. The table shows the averaged correlation and standard deviation of the EDR frequency and direct respiratory frequency measures on the 24 subjects.

\begin{tabular}{|c|c|c|c|c|}
\hline \multirow{2}{*}{} & \multicolumn{3}{|c|}{ EDR Frequency } \\
\cline { 3 - 5 } \multicolumn{2}{|c|}{} & RWD & RWA & QRS area \\
\hline \multirow{3}{*}{$\begin{array}{c}\text { Respiration } \\
\text { Frequency }\end{array}$} & Flow & $0.88 \pm 0.07$ & $0.90 \pm 0.09$ & $0.90 \pm 0.07$ \\
\cline { 2 - 5 } & Thorax & $0.85 \pm 0.08$ & $0.88 \pm 0.07$ & $0.87 \pm 0.08$ \\
\cline { 2 - 5 } & Abdomen & $0.90 \pm 0.06$ & $0.93 \pm 0.05$ & $0.92 \pm 0.06$ \\
\hline
\end{tabular}

The best correlation of EDR methods (with and without the elimination of the noisy data) and direct respiration frequency was with the measure of breathing effort from the abdomen area.

\section{Apnea Characterization}

The maximum peak-to-peak values during central apnea events decrease by $56 \%$ in RWD, $63 \%$ in RWA, and 65\% in QRS area, of the median peak-to-peak values of the stage containing the events. The calculations are based on the average of 246 classified central apnea events in 24 subjects.

In the same way as for central sleep apnea, the maximum peak-to-peak values during obstructive sleep apnea events decrease by 58\% in RWD, 57\% in RWA, and 59\% in QRS area, of the median peak-to-peak values of the stage containing the events. The calculations are based on the average of 552 classified obstructive sleep apnea events in 12 subjects.

The attenuation of the respiratory signals during central and obstructive sleep apnea, in EDR and thorax data from a single subject, is displayed in Figure2.

\section{Respiration during REM Stage}

In order to characterize respiration during REM sleep, we check the median and the variability of breathing rate in each sleep/wake state. The variability was calculated in terms of standard deviation of the stage. Figure 3 shows the frequency distribution of breathing per minutes during the sleep study of a single subject.

Table 2 presents the averaged median number of breathing/min of the distributed stages. Table 3 presents the averaged std value of the different stages.

Table 2. Averaged median number of breathing/min of the distributed stages, over all subjects.

\begin{tabular}{|c|c|c|c|}
\hline & REM & Non REM & Wake \\
\hline RWD & $16.6 \pm 2.3$ & $16.8 \pm 2.4$ & $16.7 \pm 3.2$ \\
\hline RWA & $16.7 \pm 2.5$ & $16.6 \pm 2.6$ & $16.5 \pm 3.4$ \\
\hline
\end{tabular}

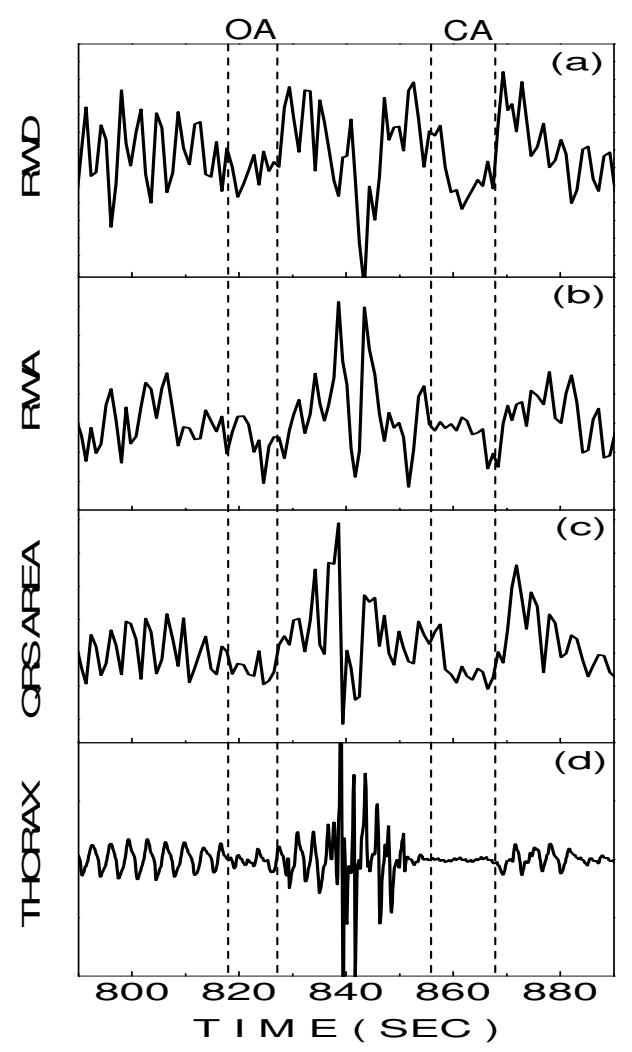

Figure 2. Central and Obstructive Sleep Apnea: (a), (b), and (c) plots contain EDR of RWD, RWA, and QRS area respectively and plot (d) shows the thorax respiration measure as a function of time (seconds) during the sleep study. Obstructive apnea occurred during the time marked by the vertical dashed line $820 \mathrm{sec}$ and it continued for 10 sec approximately. Central apnea occurred during the time marked by the vertical dashed line $860 \mathrm{sec}$ and it continuous for $12 \mathrm{sec}$ approximately. 


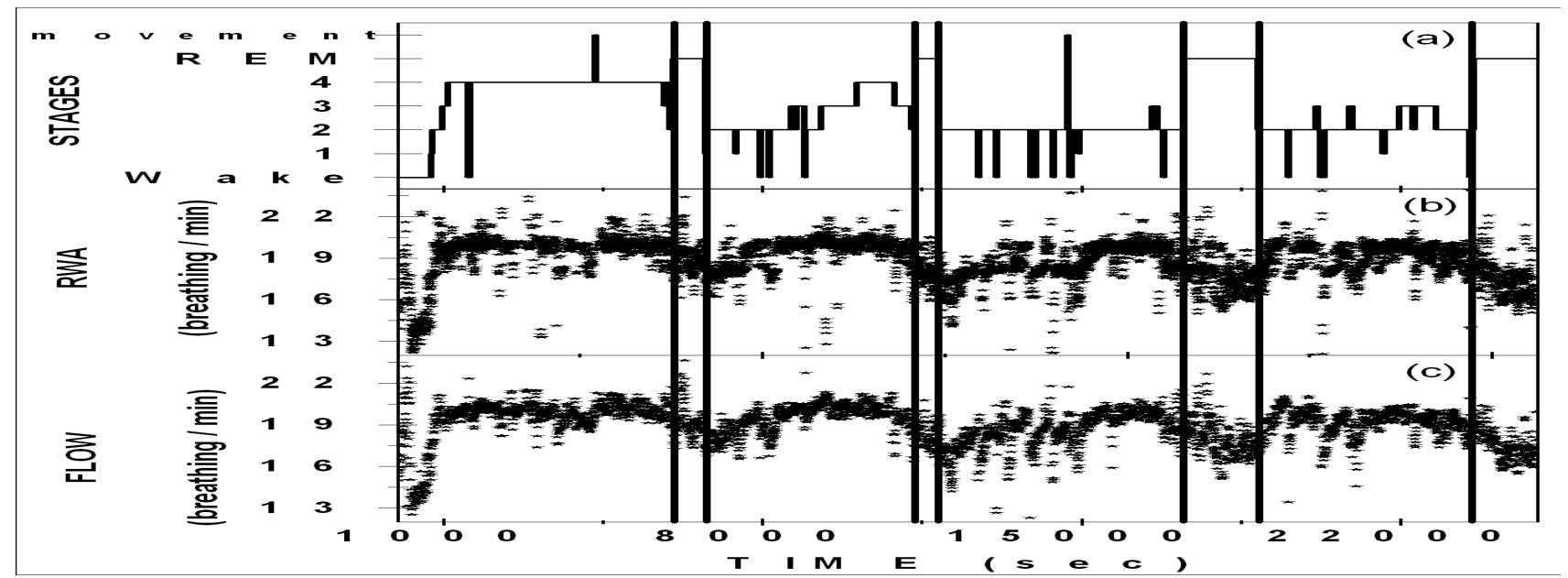

Figure 3. Frequency distribution of breathing/min during the sleep study of a single subject (a) Stages, (b) frequency based in RWA extraction and (c) Air Flow frequency calculated. The vertical lines shows the REM sleep periods. The correlation on this subject was 0.99 .

Table 3. Averaged std value of the different stages over all subjects

\begin{tabular}{|c|c|c|c|}
\hline & REM & Non REM & Wake \\
\hline RWD & $0.23 \pm 0.10$ & $0.19 \pm 0.09$ & $0.27 \pm 0.11$ \\
\hline RWA & $0.15 \pm 0.07$ & $0.13 \pm 0.06$ & $0.24 \pm 0.09$ \\
\hline
\end{tabular}

\section{Discussion and conclusions}

In this study we examined and compared three methods to calculate EDR. Our results show that the 3 methods produce similar results. We also showed that the EDR signal is best correlated with the abdominal effort signal. This finding might reflect a more pronounced motion of the abdomen wall during breathing, which affects the electrical axis of ECG recordings.

EDR can be used to extract respiratory frequency, during sleep. Respiration pauses (apneas) could be detected although tidal volume was not estimated.

The marked decrease in EDR signals during apnea events is important in characterizing obstructive apnea, and should be a sensitive feature to identify obstructive apnea. Further development is also needed in order to use EDR techniques to identify obstructive apnea.

Respiration rate is known to be approximately the same during REM and NREM sleep. Our results show no significant difference between these sleep stages. However, it is also known that during REM the respiration turns instable. This instability was observed in the dispersion of respiratory frequency during REM compared to other sleep stages (Figure 3). However, standard deviation calculation did not capture this behavior strongly. This might be due to outliers that had a substantial effect on the final results. Further work is needed in order to identify these outliers and discard them.

Our conclusion is that the EDR signals can be applied to detect and quantify respiratory activity.

\section{References}

[1] Baharav A, Shinar Z, Sivan Y, Toledo E, Keselbrenner L, Akselrod S. Autonomic Changes Associated With Sleep Onset Investigated by Time Frequency Decomposition of Heart Rate Variability. Sleep 1998, 21(3 suppl):208.

[2] Baharav A, Kotagal S, Gibbons V, Rubin BK, Pratt G, Karin J, Akselrod S. Fluctuations in Autonomic Nervous Activity during Sleep Displayed by Power Spectrum Analysis of the Heart Rate Variability. Neurology 1995, 45, pp1183-1187.

[3] Otzenberger H, Gronfier C, Simon C, Charloux A, Ehrhart J, Piquard F, Brandenbreger G. Dynamic Heart Rate Variability: A Tool for Exploring Sympathovagal Balance Continuously during Sleep in Men. Am J Physiol. 1998, 275(3 Pt 2), pp946-950.

[4] Moody GB, Mark RG, Zoccola A, Mantero S. Derivation of Respiratory Signals from Multi-Lead ECGs. Comput. Cardiol. 1985, 12.pp113-116.

[5] Rechtschaffen, A. and Kales, A. A Manual of Standardized Terminology, Techniques and Scoring System for Sleep Stages of Human Subjects. NIH publication 204. 1968. Washington D.C., US Government Printing Office. Ref Type: Generic.

[6] Shinar Z, Baharav A, Akselrod S. Detection of Different Recumbent Body Positions from the Electrocardiogram. Med. Biol. Eng. Comput. 2003, 41.pp206-210.

Address for correspondence Gabriela Dorfman Furman.

Address: Physics Dept, Tel-Aviv University, Tel-Aviv, Israel Email: gabidf@post.tau.ac.il 